\title{
Study of retinal nerve fibre layer thickness in eyes with high tension glaucoma and hemifield defect
}

\author{
Michael S Kook, Kyung-rim Sung, Soontae Kim, Ryuhwa Park, Weechang Kang
}

\begin{abstract}
Aim-To quantitatively evaluate retinal nerve fibre layer (RNFL) difference in areas of apparently normal appearing visual field in eyes with high tension glaucoma (HTG) and hemifield defects using scanning laser polarimetry.

Methods-40 eyes from 40 patients with HTG with superior or inferior hemifield defects based on the Humphrey field analyser (HFA) underwent RNFL thickness measurements. 20 normal eyes from 20 subjects matched in age and refractive error were selected as a control group. The RNFL thickness was measured with a scanning laser polarimeter. Mean RNFL thickness was evaluated in four quadrants (superior, inferior, nasal, and temporal). A superior or inferior quadrant in the defined ring of scanning laser polarimetry corresponds to inferior or superior hemifield in HFA.
\end{abstract}

Results-The mean RNFL thickness in the unaffected quadrant (the quadrant corresponding to the hemifield with apparently normal visual field based on HFA) of the HTG group was significantly thinner than the average RNFL thickness of the corresponding quadrant of the control eyes. The RNFL thickness of the unaffected quadrant in the eyes with HTG was reduced and statistically similar to that of the affected quadrant. Symmetry, calculated as the ratio of superior to inferior RNFL thickness, showed no statistical difference between the study and control group.

Conclusion-Changes in RNFL are present in the apparently normal hemifield in the eyes with HTG. The thickness of the RNFL is reduced symmetrically in both superior and inferior quadrants based on the GDx parameters.

(Br f Ophthalmol 2001;85:1167-1170)

Glaucoma is a progressive optic neuropathy with gradual loss of nerve fibre in both local and diffuse patterns. The pattern of retinal nerve fibre layer (RNFL) defects in high tension glaucoma (HTG) may differ from that of normal tension glaucoma. It has been postulated that glaucoma patients with high intraocular pressure (IOP) tend to have diffuse structural and functional damage. ${ }^{1-5}$ However, the existence of diffuse loss based on visual field in HTG has been challenged or even denied. ${ }^{\circ}$

The introduction of scanning laser polarimetry has made it possible to objectively evaluate
RNFL thickness. In previous reports, it has shown accurate and reproducible results. ${ }^{7-11}$ However, several recent reports have addressed that the results should be interpreted with caution, since scanning laser polarimetry incorporates a proprietary anterior segment compensator device that neutralises the polarisation effects of the cornea and assumes that all individuals have a fixed corneal polarisation axis of 15 degrees nasally downward. The distribution of the variable axis among individuals can lead to incomplete compensation of corneal birefringence and posterior retardation increases with increasing corneal polarisation axis. ${ }^{12}$ It is, however, important to recognise that reproducibility and the capacity of this technology to detect longitudinal changes in RNFL are not affected. Nor would it be expected to affect peripapillary RNFL retardation in a focal or geographic manner with variable corneal polarisation axis among individuals.

In this study, we used scanning laser polarimetry to quantitatively evaluate retinal nerve fibre thickness including the areas of normal appearing visual field in eyes with HTG with early visual field defects localised to a single hemifield.

\section{Patients and methods}

From August 1999 to July 2000, we prospectively evaluated one eye of 40 patients with POAG. For inclusion, patients had to meet the following criteria: (1) a mean intraocular pressure (IOP) before treatment of $22 \mathrm{~mm} \mathrm{Hg}$ or higher based on three measurements on different days; (2) normal anterior chamber angles on slit lamp biomicroscopy and gonioscopy in both eyes; (3) typical glaucomatous optic nerve appearance; (4) visual acuity of 20/30 or better; (5) glaucoma hemifield defects as detected in two consecutive fields at least 2 month apart by Humphrey field analyser (Zeiss-Humphrey, San Leandro, CA, USA) using program 30-2, standard, full threshold strategy.

The definition of glaucoma hemifield defect included the following: (1) three or more adjacent points with $\mathrm{p}<0.05$ in a total deviation (TD) probability map, or two or more test points with $<0.02$ or smaller in a TD probability map in a superior or an inferior hemifield; (2) the hemifield of the other side had no such probability symbols on both the TD and pattern deviation map; (3) a glaucoma hemifield test (GHT) result that was outside normal limits; and (4) fixation loss of less than 20\% and false positive and negative rates of less than $15 \%$. We excluded patients with a history of laser surgery, intraocular surgery in either eye, intracranial abnormalities, or a lesion upon neurological examination. 
The average mean deviation (MD) and the corrected pattern standard deviation (CPSD) as measured by the Humphrey field analyser at the second visits was -6.27 (SD 3.38) $\mathrm{dB}$ and 6.68 (4.80) dB, respectively. To further quantify the nature of the vertical hemifield asymmetry, the number of locations that met a total deviation probability value in second visual field test as defined above was counted. The mean number of abnormal probability symbol points in a TD probability map on a superior or an inferior hemifield was 18 , with range of $2-37$ in the affected hemifield (median $=17$ ).

Twenty healthy volunteers were included in this study as the control group. All subjects had best corrected visual acuity of 20/30 or better. The normal appearance of the optic nerve head in both eyes was confirmed by ophthalmological examination, and no other significant ocular diseases except for mild, age related cataract were noted by routine ophthalmological examination. None of the control group had a history of IOP elevation or ocular trauma.

All control subjects underwent visual field testing with a Humphrey field analyser using program central 24-2, standard, full threshold strategy to confirm the presence of normal visual fields. A visual field was defined as normal when the GHT result was within normal limits and the field did not meet the following criteria for visual field defect; (1) three or more adjacent points with $\mathrm{p}<0.05$ in a total deviation probability map; or (2) two or more adjacent points with $\mathrm{p}<0.02$ in a total deviation probability map. The average MD and CSPD in the control group was -0.84 (1.24) dB and $1.51(0.54) \mathrm{dB}$, respectively. All control subjects had IOP less than $22 \mathrm{~mm} \mathrm{Hg}$ using a Goldmann applanation tonometer. Normal subjects were selected to match study patients with HTG by age (SD 5 years) and refractive error (SD 2 dioptres).

There were 40 patients with HTG and 20 normal control subjects in this study. If both eyes met the inclusion criteria, one eye was randomly selected. The remaining demographic data of both study and control group are described in Table 1. The study protocol was approved by the ethics review committee of the Asan Medical Center, Ulsan University College of Medicine. Informed consent was approved by the institutional review board for human research of the Asan Medical Center and obtained from all participants.

Scanning laser polarimetry was performed on eyes that were undilated. The technique and image acquisition have been previously described. ${ }^{1011}{ }^{13}$ In brief, a polarised $780 \mathrm{~nm}$

Table 1 Demographics (mean (SD))

\begin{tabular}{llll}
\hline & HTG & Control & $p$ Value \\
\hline $\mathrm{M} / \mathrm{F}^{\star}$ & $30 / 10$ & $12 / 8$ & \\
Age & $58.77(12.35)$ & $53.6(07.34)$ & $>0.05$ \\
$\quad$ Range & $(29-77)$ & $(29-75)$ & $>0.05$ \\
Spherical equivalent & $-0.36(0.95)$ & $-0.24(1.72)$ & $<0.05$ \\
Mean deviation & $-6.27(3.38)$ & $-0.84(1.24)$ & $<0.05$ \\
Corrected pattern standard deviation & $6.68(4.80)$ & $1.51(0.54)$ & \\
\hline
\end{tabular}

^Number. diode laser illuminating light source is directed at the retina and the birefringent nerve fibre layer. Changes in the polarisation state of the reflected laser light are quantitated by a detection unit. An anterior segment compensating device is used to neutralise the polarisation originating from cornea and lens. The retardation of polarised light is measured at 65536 pixels in 0.7 seconds. An ellipse is placed around the optic nerve. This results in a measurement ellipse in the peripapillary retina. Three well centred images with good resolution were combined to form a mean composite with an average deviation of less than $8 \mu \mathrm{m}$. The sectors of the peripapillary area were divided into superior and inferior segments of 120 degrees each, a nasal segment of 70 degrees, and a temporal segment of 50 degrees. Two trained examiners (RHP, STK) performed the measurements.

Mean RNFL thickness value for average of all quadrants, superior and inferior quadrants along with superior and inferior ratio (for the ratio of superior and inferior to temporal RNFL thickness), and superior nasal ratio (for the ratio superior to nasal RNFL thickness) were calculated. Ratios were obtained to calculate relative RNFL thickness ratios to standardise measurements and reduce the influence of the optic disc size and the variability of intensity adjustments by the examiners. In addition, symmetry, defined as the ratio of superior to inferior quadrant RNFL thickness, and affected ratio, defined as the ratio of the affected quadrant (the quadrant within the peripapillary retina corresponding to the hemifield with visual field defects) to the temporal quadrant were calculated and compared with those of the normal controls. The image was then analysed with standard software (GDx, Laser Diagnostic Technologies, San Diego, CA, USA). The GDx glaucoma scanning system has a normative database, which was developed by collecting data from the normal eyes of individuals of different races. Results include comparisons of obtained images with those held in the database and statistical analysis as follows: (1) within normal limit ( $\mathrm{p}>10 \%)$; (2) outside normal limit $(\mathrm{p}<5 \%)$; or (3) borderline $(5 \%<\mathrm{p}<10 \%)$.

\section{Results}

The quality of the images was satisfactory both in the control eyes and in the eyes with HTG. Out of 40 eyes with HTG, 23 eyes had a superior hemifield defect and 17 eyes had an inferior hemifield defect. The mean RNFL thickness in the unaffected quadrant was 66.6 (SD 16.7) $\mu \mathrm{m}$ with a range of 39-101 $\mu \mathrm{m}$ while the mean RNFL thickness in the affected quadrant corresponding with the hemifield defect in HFA was 64.8 (17.3) $\mu \mathrm{m}$, with a range of 38-101 $\mu \mathrm{m}$. There was no statistically significant difference in the RNFL thickness between the unaffected and affected quadrants (paired $t$ test, $\mathrm{p}=0.63$ ).

The percentage of eyes that were outside normal limits based on the normative database in GDx software in the HTG and normal control groups along with $\mathrm{p}$ values is shown in 
Table 2 The percentage of eye which was "outside normal limits" in GDx analysis

\begin{tabular}{llrl}
\hline & POAG & Control & p Value $\left(\chi^{2}\right)$ \\
\hline Ratio of superior to inferior quadrants (symmetry index) & 17.5 & 20 & 0.536 \\
Average thickness & 32.5 & 5 & 0.015 \\
Ratio of superior to temporal quadrants (superior ratio) & 37.5 & 10 & 0.023 \\
Ratio of superior to temporal quadrants (inferior ratio) & 30.9 & 5 & 0.024 \\
Ratio of superior to nasl quadrants (superior nasal ratio) & 70.1 & 5 & 0.003 \\
Thickness of superior quadrant (superior thickness) & 40 & 5 & 0.004 \\
Thickness of superior quadrant (inferior thickness) & 30 & 5 & 0.021 \\
\hline
\end{tabular}

Table 3 Comparison of superior, inferior ratio, and superior nasal ratio between HTG and control groups (mean (SD))

\begin{tabular}{lccc}
\hline & HTG & Control & p Value \\
\hline Superior ratio & $1.46(0.41)$ & $2.14(0.33)$ & 0.0000 \\
Superior thickness & $63.95(15.55)$ & $78.7(13.00)$ & 0.0000 \\
Inferior ratio & $1.72(0.28)$ & $2.18(0.32)$ & 0.0005 \\
Inferior thickness & $67.33(18.28)$ & $79.35(8.17)$ & 0.0000 \\
Superior nasal ratio & $1.48(0.16)$ & $1.91(0.31)$ & 0.0000 \\
Affected ratio & $1.63(0.27)$ & $2.24(0.28)$ & 0.0021 \\
Unaffected ratio & $1.71(0.25)$ & $2.07(0.33)$ & 0.0014 \\
\hline
\end{tabular}

Table 2. The mean ratio of RNFL thickness in the unaffected quadrant (the quadrant within the peripapillary retina corresponding to hemifield with an apparently normal visual field) to that in the temporal quadrant was $1.71(0.25)$ while mean ratio of RNFL thickness in the affected quadrant to that in the temporal quadrant was $1.63(0.27)$. This difference was also not statistically significant (paired $t$ test, $\mathrm{p}=0.17$ ) (Table 3).

In the control group, mean ratios of RNFL in the matched unaffected quadrant to that in the temporal quadrant were $2.07(0.33)$ and $2.24(0.28)$ for the affected quadrant, respectively. These differences in both affected and unaffected quadrants between the study and control groups in mean ratios were statistically significant (1.71 $v$ 2.07: unaffected, $1.63 v$ 2.24: affected) (unpaired $t$ test, $\mathrm{p}=0.0014$ and 0.0021) (Table 3).

Symmetry, calculated as the ratio of superior to inferior RNFL thickness, was $1.00(0.14)$ in HTG, while it was $0.95(0.08)$ in the control eyes. There was no statistically significant difference between two groups (unpaired $t$ test, $\mathrm{p}=0.07)$. The mean RNFL thickness of all quadrants in the study group was 59.0 (13.6) $\mu \mathrm{m}$ and significantly lower than the control group $(64.9(8.0) \mu \mathrm{m}) \quad(\mathrm{p}=0.04$, unpaired $t$ test). RNFL thickness in the superior and inferior quadrants was significantly thinner in the study group than in the control group. Both superior and inferior ratios along with superior nasal ratio also showed significant differences between the study and the control groups $(\mathrm{p}<0.001$, Table 3).

\section{Discussion}

Previous clinical studies have suggested that patients with HTG show a mostly diffuse type of glaucomatous optic nerve damage. ${ }^{1-4} \mathrm{Re}-$ cently, Kubota et al, using scanning laser polarimetry, compared the measurements of symmetry, calculated as a ratio of superior to inferior RNFL thickness, in patients with HTG with eyes with NTG and found that more localised defects on the inferior RNFL were noted in eyes with NTG $(1.00 v 1.18) .{ }^{14}$ However, they did not describe the pattern of visual field loss in both groups other than the average mean deviation (MD) $(-6.7(6.8) \mathrm{dB} v$ $-4.7(5.9) \mathrm{dB}$ ) and the corrected pattern standard deviation (CPSD) (5.6 (4.1) $v 4.1$ (3.9)) as measured by the Humphrey field analyser.

Jonas and Hayreh showed, in an experimental model of chronic high pressure glaucoma using rhesus monkeys, at least a mixture of localised and diffuse patterns of optic nerve damage, contradicting the concept that a mostly diffuse type of optic neuropathy occurs in high pressure glaucoma. ${ }^{6}$ They also speculated that all glaucomatous, optic nerve fibre loss might occur in a localised pattern that may be too small to be visible at an early stage and may only become visible later when many of them combine with disease progression. As a result, diffuse retinal nerve fibre layer loss in eyes with very advanced glaucomatous optic nerve damage may be the result of broadening localised retinal nerve fibre layer defects.

In this study we used scanning laser polarimetry to quantitatively evaluate the status of RNFL in the areas of apparently normal hemifield and a pattern of RNFL loss in eyes with high tension glaucoma that had early glaucomatous visual field defect confined to one hemifield. The RNFL thickness in the unaffected quadrant showed a statistically similar reduction as in the affected quadrant. This study also revealed that the RNFL was significantly thinner in the unaffected as well as the affected quadrants of eyes with glaucoma compared with the corresponding areas of normal control eyes. These findings suggest that diffuse NFL change may be present even in the apparently normal hemifield, based on HFA in patients with high tension glaucoma.

Symmetry was not different statistically $(p>0.05)$ between patients with high tension glaucoma (1.00) and the controls (0.95). In order to standardise interindividual variability in the RNFL thickness, the mean ratios of RNFL thickness in both affected and unaffected quadrants and in temporal quadrants were evaluated and showed significant reductions compared with those of corresponding quadrants in the control group. These results may also confirm generalised RNFL loss and indicate that conventional white on white perimetry failed to show glaucomatous defects in the unaffected hemifield despite the diffuse NFL loss noted in both the superior and inferior quadrants. Therefore, glaucomatous eyes with hemifield defects on conventional perimetry are at risk for development of visual field defects in apparently normal areas.

Visual field loss in glaucoma has been classified into localised and diffuse types. ${ }^{15}$ Localised loss includes arcuate and paracentral defects as seen in our patients. In contrast, diffuse loss has been defined as a reduction in retinal sensitivity over the visual field typically seen as a contraction of isoptres in kinetic perimetry or reduced retinal sensitivity in static threshold perimetry. Despite localised visual field loss confined to one hemified in our study, diffuse RNFL changes were found based on scanning 
laser polarimetry. Our results may be in agreement with findings by Jonas and Hayreh that purely localised visual field loss in high pressure glaucoma is, in fact, a mixture of a localised and a diffuse pattern of optic nerve damage. There might have been a "merging" of numerous localised nerve fibre losses, worse in the affected quadrant.

We must also consider that perimetry assesses visual function, whereas NFA GDx evaluates the anatomical structures. And these two entities are not identical. Airaksinen and Drance have reported that there is a substantial amount of generalised loss of nerve fibres without measurable effect on the retinal sensitivity. ${ }^{16}$ However, after a certain level of diffuse structural changes has been reached, further loss in RNFL is associated with an increasingly steep reduction in retinal sensitivity. Thus, it seems that the small additional nerve fibre loss in the affected quadrant might have accounted for the corresponding hemifield defect and this nerve fibre loss has not been detected by the parameters of NFA GDx, such as symmetry.

In summary, our study revealed that diffuse RNFL changes were present throughout retinas including the hemifield with no apparent visual field defect by conventional perimetry in eyes with HTG, and these changes were detected quantitatively using the NFA GDx. In high tension glaucoma, existence of both localised and diffuse nerve fibre loss may explain the pattern of diffuse RNFL changes by NFA GDx manifesting as localised visual field defect confined to one hemifield as noted in our study. This localised RNFL loss beyond the structural threshold may present as hemifield loss in conventional perimetry in the affected quadrant and may not be detected by the NFA GDX parameters. More sensitive measures are needed in NFA GDx to detect these localised changes. In addition, conventional white on white automated perimetry, as used in our study, may not be sensitive enough to detect the functional damage in the apparently normal hemifield and more sensitive tools in the future may better correlate with the RNFL loss noted by scanning laser polarimetry.

Presented in part at the 3rd International Glaucoma Symposium, Prague, Czech Republic, 21-25 March 2001.

1 Spaeth GL, Hitchings RA, Sivalingam E. The optic disc in glaucoma: pathogenetic correlation of five patterns of cupping in chronic open-angle glaucoma. Trans Am Acad Ophthalmol Otolaryngol 1976;181:217-23.

2 Drance SM, Airaksinen PJ, Price M, et al. The correlation of functional and structural measurements in glaucoma patients and normal subjects. Am f Ophthalmol 1986;102: 612-6.

3 Geijssen HC, Greve EL. Focal ischaemic normal pressure glaucoma versus high pressure glaucoma. Doc Ophthalmol 1990;75:291-301.

4 Caprioli J. Correlation between disc appearance and type of glaucoma. In: Varma R, Spaeth GL, eds. The optic nerve in glaucoma. Philadephia: Lippincott, 1993:91-8.

glaucoma. Philadephia: Lippincott, 1993:91-8.
5 Balwantray CC, Drance SM, Douglas GR, et al. Visual field Balwantray CC, Drance SM, Douglas GR, et al. Visual field f Ophthalmol 1989;108:636-642.

6 Jonas JB, Hayreh SS. Localised retinal nerve fibre layer defects in chronic experimental high pressure glaucoma in rhesus monkey. Br f Ophthalmol 1999;83:1291-1295.

7 Weinreb RN, Shakiba S, Zangwill L. Scanning laser polarimeter to measure the nerve fiber layer of normal and glaucomatous eyes. Am f Ophthalmol 1995;119:627-636.

8 Niessen AGJE, Van den Berg TJTP, Langerhorst CT, et al. Retinal nerve fiber layer assessment by scanning laser polarimeter and standardized photography. Am F Ophthalmol 1996;121:484-493.

9 Weinreb RN, Shakiba S, Sample PA, et al. Association between quantitative nerve fiber layer measurement and visual field loss in glaucoma. Am $\mathcal{F}$ Ophthalmol 1995;120: 732-738.

10 Zangwill L, Berry CA, Garden VS, et al. Reproducibility of retardation measurements with the nerve fiber analyzer II. retardation measurements with

11 Tjon-Fo-Sang MJH, van Strik R, de Vries J, et al. Improved reproducibility of measurements with the nerve fiber reproducibility of measurements with

12 Greenfield DS, Knighton RW, Huang XR. Effect of corneal polarization axis on assessment of retinal nerve fiber layer thickness by scanning laser polarimetry. Am $\mathcal{F}$ Ophthalmol 2000;129:715-722.

13 Kogure S, Chiba T, Kinoshita T, et al. Effects of artefacts on scanning laser polarimetry on retinal nerve fibre layer thickness measurement. Br f Ophthalmol 2000;84:10131017 .

14 Kubota T, Khalil AK, Honda M, et al. Comparative study of retinal nerve fiber layer damage in Japanese patients with normal- and high-tension glaucoma. F Glaucoma 1999;8: 363-366.

15 Henson DB, Artes PH, Chauhan BC. Diffuse loss of sensitivity in early glaucoma. Invest Ophthalmol Vis Sci 1999;40: tivity in ear 51 .

16 Airaksinen PJ, Drance SM, Douglas GR, et al. Visual field and retinal nerve fiber layer comparisons in glaucoma. Arch Ophthalmol 1985;103:205-7. 